\title{
ABSI is a poor predictor of insulin resistance in Chinese adults and elderly without diabetes
}

Kai $\mathrm{Wu}^{1,2,3 *}$, Sen $\mathrm{He}^{1 *}$, Yi Zheng ${ }^{1 *}$, Xiaoping Chen'

\begin{abstract}
Objective: Recently, a new obesity index (A Body Shape Index, ABSI) based on waist circumference (WC) was developed, and high ABSI corresponds to a more central concentration of body volume. It is well known that central obesity is closely linked with insulin resistance (IR). Therefore, our study aimed to examine the discriminatory power of $A B S I$ for IR in Chinese adults and elderly without diabetes. Subjects and methods: In 2007, a cross-sectional study was made. In this study, 570 individuals without diabetes were available for analysis (male: $56.1 \%$, mean age: $62.3 \pm 6.5$ years). Insulin resistance was assessed by homeostasis model assessment (HOMA-IR). Areas under the receiver operating characteristic $(\mathrm{ROC})$ curves were determined to identify variables/models that could predict insulin resistance. Results: $A B S I$ was associated with IR, the cut-off points was 0.0785 $\mathrm{m} 11 / 6 \mathrm{~kg}-2 / 3$ to identifying IR and the area under the ROC (AUC) curve was 0.618 (95\% $\mathrm{Cl}: 0.561-0.675)$, which was not better than body mass index BMI (AUC $=0.753 ; 95 \% \mathrm{Cl}: 0.706-0.801$ ), WC (AUC = 0.749; $95 \% \mathrm{Cl}: 0.700-0.797$ ), and fasting plasma glucose (FPG, $\mathrm{AUC}=0.752 ; 95 \% \mathrm{Cl}: 0.705-0.799$ ). Furthermore, combination with $A B S I$ could improve the discriminatory power of other variables for IR. The AUC curve increased from 0.753 to 0.771 for $\mathrm{BMI}, 0.749$ to 0.754 for WC, 0.752 to 0.769 for FPG, respectively. Conclusions: $A B S I$ is associated with IR in the general Chinese adults and elderly without diabetes, but the discriminatory power for IR is poor. It is recommended that ABSI be used in combination with other variables. Arch Endocrinol Metab. 2018;62(5):523-9
\end{abstract}

Keywords

Body shape index; body mass index; waist circumstance; insulin resistance
${ }^{1}$ Department of Cardiovascular Medicine, West China Hospital, Sichuan University, Chengdu, China 2 Department of Cardiovascular Medicine, Guangyuan Central Hospital, Guangyuan, China ${ }^{3}$ Department of Cardiovascular Medicine, 903 Hospital, and Center for Medical Radiation Biology, Institute of Materials, China Academy of Engineering Physics, Mianyang, China

\# Kai Wu, Sen He and Yi Zheng equally contributed to the article

\section{Correspondence to:}

Xiaoping Chen

Department of Cardiovascular

Medicine,

West China Hospital

Sichuan University,

Chengdu 610041, China

cxpdewenzang@163.com

Received on April/2/2018 Accepted on Jun/13/2018

DOI: $10.20945 / 2359-3997000000072$

\section{INTRODUCTION}

$\mathrm{I}_{\mathrm{i}}^{\mathrm{n}}$ nsulin resistance is characterized by a decrease in the ability of insulin to stimulate the use of glucose by muscles and adipose tissues and to suppress hepatic glucose production and output (1). Insulin resistance plays a patho-physiological role in type 2 diabetes and metabolic disorders, and is frequently present in hypertension, coronary artery disease, cancer, endothelial dysfunction and depression (2-6). Early identification of insulin resistant individuals is important for managing the health problems associated with insulin resistance.

It is well known that obesity is closely linked with insulin resistance (7), and many studies have shown that obesity indexes, such as body mass index (BMI) and waist circumstance (WC), could estimate insulin resistance (8-11). Recently, Krakauer and Krakauer (12) developed a new obesity index, namely a body shape index (ABSI), based on WC that is approximately independent of height, weight, and BMI. High ABSI indicates that $\mathrm{WC}$ is higher than expected for a given height and weight and corresponds to a more central concentration of body volume (12). Krakauer and cols. $(12,13)$ have shown that ABSI could predict mortality in the general American and British population, even better than BMI and WC. In consideration of the aforementioned characteristics of ABSI and the present findings $(12,13)$, we conclude that ABSI might potentially be a good marker of insulin resistance. Since ABSI was developed, it has led to substantial international interests (14-22). However, to the best of our knowledge, the specific relationship between ABSI and insulin resistance about Chinese adults and elderly was not studied previously, only some foreign studies were reported (23-25). Therefore, the aims of our study were to examine the discriminatory power of ABSI for insulin resistance in Chinese adults and elderly without diabetes. 


\section{SUBJECTS AND METHODS}

\section{Study population}

In 2007, a cross-sectional study was conducted among 711 adults and elderly in an urban community located in Chengdu, Sichuan province, China. The study was supported by mega-projects of science research for the 11th five-year plan, China (Trends in the incidence of metabolic syndrome and integrated control in China). Among the 711 individuals, 141 of them had no data about insulin or were diagnosed with diabetes. Therefore, the remaining 570 individuals were available for analysis (male: $56.1 \%$, mean age: $62.3 \pm 6.5$ years). The study was approved by Ministry of Health of China, as well as by the Ethics Committee of West China Hospital of Sichuan University. All participants gave informed consent.

\section{Data collection}

Anthropometric measurements, such as height, weight and WC, were conducted at the time of interview. Height was measured using a digital stadiometer with a fixed vertical backboard and an adjustable head piece. Weight was measured on a digital scale. At the end of a normal exhalation, WC was measured to the midpoint between the lower border of the rib cage and the iliac crest. Blood samples were drawn from the antecubital vein in the morning after a 12 -h fasting. Laboratory tests included fasting plasma glucose (FPG), lowdensity lipoprotein cholesterol (LDL-C), high-density lipoprotein cholesterol (HDL-C), triglyceride (TG) and insulin. FPG, LDL-C, HDL-C and TG were measured enzymatically using a MODULAR P800 Analyzer (Roche Diagnostics). Fasting serum insulin was measured by radioimmunoassay (XH-6010, Xi'an, China). These chemistries were measured at the laboratory of West China Hospital (Chengdu, China).

\section{Related definitions}

Insulin resistance was assessed by homeostasis model assessment (HOMA-IR), which was calculated according to the following formula: fasting insulin $(\mathrm{mU} / \mathrm{mL}) \times$ fasting glucose $(\mathrm{mmol} / \mathrm{L}) / 22.5(8)$. Insulin resistance was defined as being in the highest quintile of HOMA score $(\geq 1.66)$, according to the previous studies $(26,27)$. BMI was calculated as weight in $\mathrm{kg} /$ height in $\mathrm{m}^{2}$. ABSI was defined as $\mathrm{WC} /\left(\mathrm{BMI}^{2 / 3}\right.$ height $\left.^{1 / 2}\right)$, expressing $\mathrm{WC}$ and height in $\mathrm{m}(12)$. Those with hypertension were defined as having systolic blood pressure of at least $140 \mathrm{mmHg}$ and/or diastolic blood pressure of at least $90 \mathrm{mmHg}$ and/or currently taking antihypertensive medications. Diabetes mellitus was defined as one of the following at follow-up assessment: (1) fasting plasma glucose $\geq 7.0$ $\mathrm{mmol} / \mathrm{L} ;(2)$ a positive response to the question, "Has a doctor ever told you that you have diabetes?"or (3) current use of insulin or oral hypoglycemic agents (14).

\section{Statistical analysis}

Descriptive statistics (mean \pm standard deviation, median + inter-quartile, percentages, etc.) were used to summarize demographic and metabolic characteristics. Correlations for normally distributed and skewed variables were assessed, respectively, by Pearson and Spearman correlation analysis. Krakauer and cols. (12) thought that ABSI expressed the excess mortality risk from high WC that was complementary to BMI and to other known risk factors. We also estimated whether combination with ABSI could improve the discriminatory power of other variables for insulin resistance. To estimate whether combination with ABSI could improve the discriminatory power of other variables for insulin resistance, different logistic regression models were developed. Areas under the receiver operating characteristic (ROC) curves were used to estimate the discriminatory power of each variable/model for insulin resistance. We use Hosmer and Lemeshow test to estimated whether combination with ABSI could improve the discriminatory power of other variables for insulin resistance. The statistic follows the chi-square distribution and a larger $\mathrm{p}$ value indicates the model fit better. In view of the influence of age and sex as variables, we run the analyses with the adjustment of age and sex.

We also set the optimal cut-off point, which represents the optimal combination of sensitivity and specificity for the study sample. For statistical analysis, SPSS software was used (version 17.0; SPSS, Chicago, IL), and statistical significance was defined as $\mathrm{p}<0.05$.

\section{RESULTS}

\section{Demographic and metabolic characteristics}

The demographic and metabolic characteristics of the 570 subjects (male: $56.1 \%$, mean total age: $62.3 \pm 6.5$ years, mean male age: $63.3 \pm 6.2$ years, mean female age:61.0 \pm 6.7 ) are provided in Table 1 according to gender. The mean total values were $23.5 \pm 3.2 \mathrm{~kg} /$ 
$\mathrm{m}^{2}, 82.0 \pm 9.6 \mathrm{~cm}, 0.0786 \pm 0.0047 \mathrm{mll} / 6 \mathrm{~kg}-2 / 3$ and $4.6 \pm 0.7 \mathrm{mmol} / \mathrm{L}$ for BMI, WC, ABSI and FPG respectively, the mean male values were $23.4 \pm$ $3.0 \mathrm{~kg} / \mathrm{m}^{2}, 83.6 \pm 9.3 \mathrm{~cm}, 0.0792 \pm 0.0046 \mathrm{~m}^{11 / 6} \mathrm{~kg}^{-2 / 3}$ and $4.6 \pm 0.8 \mathrm{mmol} / \mathrm{L}$, and the mean female values were $23.6 \pm 3.4 \mathrm{~kg} / \mathrm{m}^{2}, 80.0 \pm 9.7 \mathrm{~cm}, 0.0778 \pm$ $0.0048 \mathrm{~m}^{11 / 6} \mathrm{~kg}^{-2 / 3}$ and $4.6 \pm 0.7 \mathrm{mmol} / \mathrm{L}$. Other variables are shown in Table l. For age, SBP, DBP, HDL-C, insulin, HOMA, height, weight, and ABSI, there is statistically significant difference between male and female, and all the $\mathrm{P}$ values were less than 0.001 . But for the other variables, there is no significant difference between male and female. Logistic regression analysis showed that ABSI, BMI, WC and FPG were independently associated with insulin resistance, with adjusted OR values 2.395 (95\%CI: $1.358-4.225$, p < $0.003), 1.357$ (95\%CI: 1.232-1.496, p < 0.001), 1.117 (95\%CI: 1.081-1.154, $\mathrm{p}<0.001)$ and 3.853 (95\%CI: 2.557-5.807, $\mathrm{p}<0.001)$ respectively. Correlation coefficients of ABSI with WC, BMI, height and weight were $0.621(\mathrm{p}<0.001), 0.121(\mathrm{p}=0.004), 0.168(\mathrm{p}<$ $0.001)$ and $0.180(\mathrm{p}<0.001)$, respectively.

Areas under receiver operating characteristic curves for potential variables/models identifying insulin resistance

Table 2 presents the areas under the ROC curves identifying insulin resistance from potential variables. Among the anthropometric variables (BMI, WC, ABSI), the ROC curve analyses showed that ABSI had the poorest discriminatory power for insulin resistance,

Table 1. Demographic and metabolic characteristics

\begin{tabular}{|c|c|c|c|}
\hline Variable & Total (570) & Male (320) & Female (250) \\
\hline Age (years) & $62.3 \pm 6.5$ & $63.3 \pm 6.2$ & $61.0 \pm 6.7^{\star}$ \\
\hline $\mathrm{SBP}(\mathrm{mmHg})$ & $134.7 \pm 19.1$ & $136.7 \pm 18.2$ & $132.2 \pm 19.9^{*}$ \\
\hline $\mathrm{DBP}(\mathrm{mmHg})$ & $79.4 \pm 10.1$ & $80.6 \pm 10.0$ & $77.9 \pm 10.0^{*}$ \\
\hline $\mathrm{FPG}(\mathrm{mmol} / \mathrm{L})$ & $4.6 \pm 0.7$ & $4.6 \pm 0.8$ & $4.6 \pm 0.7$ \\
\hline $\mathrm{TG}(\mathrm{mmol} / \mathrm{L})$ & $1.5(1.1,2.2)$ & $1.7(1.1,2.0)$ & $1.7(1.2,2.2)$ \\
\hline $\mathrm{HDL}-\mathrm{C}(\mathrm{mmol} / \mathrm{L})$ & $1.4(1.3,1.7)$ & $1.4(1.2,1.6)$ & $1.6(1.3,1.8)^{*}$ \\
\hline LDL-C (mmol/L) & $3.0 \pm 0.7$ & $3.0 \pm 0.8$ & $3.0 \pm 0.7$ \\
\hline Insulin (mU/L) & $5.2(3.6,7.3)$ & $4.8(3.4,6.6)$ & $5.6(4.1,8.1)^{\star}$ \\
\hline HOMA & $1.05(0.72,1.53)$ & $0.98(0.67,0.98)$ & $1.16(0.84,1.67)^{*}$ \\
\hline Height (cm) & $162.2 \pm 7.7$ & $166.7 \pm 5.9$ & $156.6 \pm 6.0^{*}$ \\
\hline Weight $(\mathrm{kg})$ & $62.0 \pm 10.1$ & $65.1 \pm 9.4$ & $58.0 \pm 9.6^{\star}$ \\
\hline $\mathrm{BMI}\left(\mathrm{kg} / \mathrm{m}^{2}\right)$ & $23.5 \pm 3.2$ & $23.4 \pm 3.0$ & $23.6 \pm 3.4$ \\
\hline WC (cm) & $82.0 \pm 9.6$ & $83.6 \pm 9.3$ & $80.0 \pm 9.7$ \\
\hline ABSI $\left(\mathrm{m}^{11 / 6} \mathrm{~kg}^{-2 / 3}\right)$ & $0.0786 \pm 0.0047$ & $0.0792 \pm 0.0046$ & $0.0778 \pm 0.0048^{*}$ \\
\hline Hypertension (\%) & $50 \%$ & 51.2 & 48.4 \\
\hline
\end{tabular}

Data are presented as means $\pm S D$, median (interquartile range), or percentage. "*” means $p<0.05$.

ABSI: a body shape index; BMl: body mass index; WC: waist circumference; SBP: systolic blood.

Table 2. Comparison of areas under receiver operating characteristic curves for potential variables identifying insulin resistance

\begin{tabular}{lcc}
\hline Variable & Area under ROC Curve & $\mathbf{9 5 \%} \mathbf{C l}$ \\
\hline Biological variable & & $0.705-0.799$ \\
FPG & 0.752 & $0.585-0.699$ \\
TG & 0.642 & $0.296-0.405$ \\
HDL-C & 0.351 & $0.467-0.585$ \\
LDL-C & 0.526 & $0.706-0.801$ \\
Anthropometric variable & & $0.700-0.797$ \\
BMl & 0.753 & $0.561-0.675$ \\
WC & 0.749 & \\
ABSI & 0.618 & \\
\hline
\end{tabular}

ROC: receiver operating characteristic; $\mathrm{Cl}$ : confidence interval. Other abbreviations as in Table 1. 
with an area under the ROC curve of 0.618 (95\%CI: 0.561-0.675) (Table 2, Figure 1). Compared with ABSI, BMI and WC had better discriminatory power, as they had areas under the ROC curves of 0.753 (95\%CI: $0.706-0.801)$ and 0.749 (95\%CI: $0.700-$ 0.797 ), respectively (Table 2, Figure 1). The areas under the ROC curves of other variables were less than 0.700 , except FPG (Table 2).

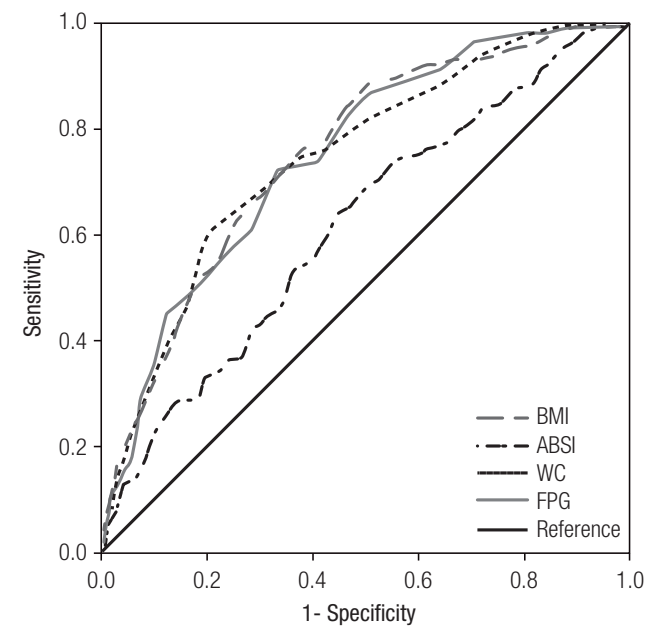

Figure 1. Receiver operating characteristic curves of BMI, WC, ABSI and FPG identifying insulin resistance.

Abbreviations as in Table 1.
We also estimated whether combination with ABSI could improve the discriminatory power of other variables for insulin resistance (Table 3). In combination with ABSI, BMI could improve the discriminatory power, and the area under the ROC curve increased from 0.753 to 0.771 (Tables 2 and 3 ). In combination with ABSI, WC and FPG could also improve the discriminatory power, and the areas under the ROC curves increased from 0.749 for WC to 0.754 in model 2, from 0.752 for FPG to 0.769 in model 3, respectively (Tables 2 and 3 ).

When considering age and sex as variables of adjustment in the analyses, we found that both age and sex affect the results as to BMI and WC, but as to ABSI and FPG, only sex alone affect the result. As to the ROC of different models, ABSI ROC curve analysis according to the sex, the male area under the curve is 0.689 , female is 0.599 , the difference is very big still.

The optimal cut-off points to identifying insulin resistance in our population were the following values: $24.2 \mathrm{~kg} / \mathrm{m}^{2}$ for BMI, $87.5 \mathrm{~cm}$ for WC, 0.0785 $\mathrm{mll} / 6 \mathrm{~kg}-2 / 3$ for ABSI, $4.75 \mathrm{mmol} / \mathrm{L}$ for $\mathrm{FPG}$, -1.26 for model $1,-1.31$ for model 2 and -1.31 for model 3, respectively; and other parameters for these optimal cut-off points are shown in Table 4.

Table 3. Comparison of areas under receiver operating characteristic curves for different models identifying insulin resistance

\begin{tabular}{lccr}
\hline Variable & Model 1 & Model 2 & Model 3 \\
\hline ABSI & $\sqrt{ }$ & $\sqrt{ }$ & $\sqrt{ }$ \\
BMI & $\sqrt{ }$ & $\sqrt{ }$ & $\sqrt{ }$ \\
WC & & & $-14.626+85.819^{*} \mathrm{ABSI}+1.343^{*} \mathrm{FPG}$ \\
FPG & $-16.029+89.151^{*} \mathrm{ABSI}+0.312^{*} \mathrm{BMl}$ & 0.091 \\
Model formula & 0.133 & 0.302 & $0.769(0.723-0.816)$ \\
Hosmer and Lemeshow test (p value) & $0.771(0.725-0.818)$ & $0.754(0.706-0.802)$ & \\
AROC (95\%Cl) & & & \\
\hline
\end{tabular}

For each model, ABSl in $\mathrm{m}^{11 / 6} \mathrm{~kg}^{2 / 3}$, TG in $\mathrm{kg} / \mathrm{m}^{2}$, WC in $\mathrm{cm}$ and FPG in mmol/L. The symbol " $/$ " meant that the specific variable was included in the specific model. Abbreviations as in Tables 1 and 2.

Table 4. Sensitivity and specificity of different variables/models for identifying insulin resistance

\begin{tabular}{lccccc}
\hline Variable/Model & Optimal cut-off point & Sensitivity (\%) & Specificity (\%) & + LR & - LR \\
\hline BMI & 24.2 & 72.2 & 66.8 & 2.17 & 0.42 \\
WC & 87.5 & 60.0 & 80.2 & 3.03 & 0.50 \\
ABSI & 0.0785 & 65.2 & 54.3 & 1.43 & 0.64 \\
FPG & 4.75 & 72.2 & 66.8 & 2.17 & 0.42 \\
Model 1 & -1.26 & 73.0 & 74.1 & 2.82 & 0.36 \\
Model 2 & -1.31 & 71.3 & 71.4 & 2.49 & 0.40 \\
Model 3 & -1.31 & 71.3 & 71.4 & 2.49 & 0.40 \\
\hline
\end{tabular}

+LR: positive likelihood ratio; -LR: negative likelihood ratio. Other abbreviations as in Table 1. 


\section{DISCUSSION}

Recently, ABSI was studied extensively, some studies declared it can predicted mortality of general people or affected incidence of obesity or metabolic disorder $(3,7,12,23)$. The aims of our study were to examine the discriminatory power of ABSI for insulin resistance in Chinese adults and elderly without diabetes. Our findings showed that ABSI is associated with insulin resistance in the general Chinese adults and elderly without diabetes, but the discriminatory power for insulin resistance is poor. Furthermore, combination with ABSI could improve the discriminatory power of other variables for IR.

Insulin resistance is associated with many health problems, such as type 2 diabetes, metabolic disorders, hypertension, coronary artery disease, cancer, endothelial dysfunction, depression and pulmonary arterial hypertension (2-6,28). As to Asian, McKeigue and cols. (29) found that insulin resistance syndrome is prevalent in South Asian populations and associated with a pronounced tendency to central obesity. It can lead to a higher prevalence of diabetes ( $19 \%$ vs $4 \%$ ), higher blood pressures, higher fasting and post-glucose serum insulin concentrations, higher plasma triglyceride, and lower HDL cholesterol concentrations. Lee and cols. (30) also found HOMA-IR could identify dysglycemia and type 2 diabetes mellitus. The direct relation between insulin resistance and fatness is well known $(3,7)$. Banerji and cols. (31) also found that increased visceral fat was related to dyslipidemia and increased frequency of insulin resistance and may account for the increased prevalence of diabetes mellitus and cardiovascular disease in Asian Indians. The underlying mechanisms of obesity inducing insulin resistance include inflammation, mitochondrial dysfunction, hyperinsulinemia and lipotoxicity, oxidative stress, genetic background, aging, fatty liver, hypoxia and lipodystrophy $(7,32)$. Many studies have shown that obesity indices, such as BMI and WC, could estimate insulin resistance (8-11), our findings confirmed these studies.

The new obesity indice, namely ABSI, is based on WC that is approximately independent of height, weight, and BMI (12). The developers of ABSI have claimed that the index is more related to visceral than peripheral fat, which indicates that ABSI might potentially be a good marker of insulin resistance. However, the present findings are opposite to the hypothesis. Currently, there is not sufficient information available for us to understand why, however, some speculations could be made. ABSI is based on an American population from NHANES 1999-2004 (mainly including Mexicans, blacks and whites) (12). The American study population of Krakauer and cols. (12) has a higher height, BMI and WC than our study population. On the other hand, Asian populations are more prone to abdominal obesity and low muscle mass compared with their Western counterparts (3335). Therefore, the coefficients of ABSI based on American population might not be suitable for other populations, especially for Asian populations. For example, Haghighatdoost and cols. (15) concluded that ABSI was a weak predictor for CVD risks and metabolic syndrome among Iranian adults, and Maessen and cols. (16) suggested that ABSI was not capable to determine cardiovascular diseases presence in the Netherlandish population (16). The authors thought that body height might confound the values of ABSI for health problems $(15,16)$. Cheung (18) showed that ABSI was less associated with incident hypertension than BMI and WC in the Indonesian population, which might be caused by the lower mean BMI and WC (18). Furthermore, the coefficients of ABSI might be influenced by the age, even if Krakauer and cols. (12) showed that ABSI correlation with mortality hazard held across the range of age. In an published article by us (14), the subjects were with a mean age of $48.1 \pm 6.2$ years, which were younger than the present population, and the correlation coefficients of ABSI with BMI, height and weight were $0.611(p<0.001),-0.040(p=0.292), 0.283$ $(\mathrm{p}<0.001)$ and $0.155(\mathrm{p}<0.001)$, respectively. The correlation coefficients did not hold across the range of age. Those might imply the coefficients of ABSI might not be suitable for other populations. Although ABSI had poor discriminatory power for insulin resistance, it could improve the discriminatory power of other variables (Table 3 ).

The present study has some limitations that should be considered. Firstly, insulin resistance is traditionally determined by euglycaemic-hyperinsulinaemic clamp technique (36), but in general population, it is more convenient and cost effective to estimate it using HOMA IR, which is an established test in epidemiological studies $(37,38)$. Secondly, insulin resistance based on HOMA IR has been defined differently in different studies. Values based on 50th percentile, 75 th percentile, 90 th percentile, lower boundary of the top quintile or tertile 
of HOMA score have been used previously (39). We defined insulin resistance arbitrarily as HOMA score greater than the 80 th percentile $(\geq 1.66)$, but this is commonly practiced $(26,27)$. Thirdly, for the absence of an oral glucose tolerance test, some individuals with diabetes might be included in the analysis, which could confound the results to some extent. Fourthly, no comparisons between different races might be another limitation. In addition, some other study considered that refined estimations of HOMA-IR levels could not exclude the indices such as age and sex (40). As to nondiabetic Spanish, there are gender-specific differences in HOMA-IR, with increased levels in women over fifty years of age that may be related with changes in body fat distribution after menopause. So, it meant that age and sex might influence the result of HOMA-IR, but our mode design did not regard sex and age as influencing factors.

Of course, the choice of statistical method is very important, because different statistical method may result in discriminating results. In our research, logistic regression analysis revealed that these variables associated with IR, and ROC suggested the identified ability. They are the common methods of clinical research.

In conclusion, our findings showed that ABSI is associated with insulin resistance in the general Chinese adults and elderly without diabetes, but the discriminatory power for insulin resistance is poor. It is recommended that ABSI be used in combination with other variables. Further studies about ethnic specificities of ABSI are needed and warranted, as well as the complementary values of ABSI to other known risk factors.

Authorship: Xiaoping Chen: guarantor of integrity of the entire study, manuscript editing, manuscript review; Kai Wu, Sen He and Yi Zheng: study concepts; Kai Wu: manuscript preparation; Sen He: study design, statistical analysis; Yi Zheng: data analysis.

All the Authors have read the manuscript and have agreed to submit it in its current form for consideration for publication in the AE\&M.

Acknowledgments: not applicable.

Funding statement: this study was supported by megaprojects of science research for China's 11th 5-year plan (grant number: 2006BAI01A01), the National Natural Science Foundation of China (grant number: 81600299) and Youth Innovation and Medical Research of Sichuan Province (grant number: Q15069).

Disclosure: no potential conflict of interest relevant to this article was reported.

\section{REFERENCES}

1. Barazzoni R, Deutz NE, Biolo G, Bischoff S, Boirie Y, Cederholm $\mathrm{T}$, et al. Carbohydrates and insulin resistance in clinical nutrition: Recommendations from the ESPEN expert group. Clin Nutr. 2017 Apr;36(2):355-63.

2. DeFronzo RA, Ferrannini E. Insulin resistance. A multifaceted syndrome responsible for NIDDM, obesity, hypertension, dyslipidemia, and atherosclerotic cardiovascular disease. Diabetes Care. 1991;14(3):173-94.

3. Sinaiko AR, Steinberger J, Moran A, Prineas RJ, Vessby B, Basu $S$, et al. Relation of body mass index and insulin resistance to cardiovascular risk factors, inflammatory factors, andoxidative stress during adolescence. Circulation. 2005;111(15):1985-91.

4. Muniyappa R, Sowers JR. Role of insulin resistance in endothelial dysfunction. Rev Endocr Metab Disord. 2013;14(1):5-12.

5. Orgel E, Mittelman SD. The links between insulin resistance, diabetes, and cancer. Curr Diab Rep. 2013;13(2):213-22.

6. Kan C, Silva N, Golden SH, Rajala U, Timonen M, Stahl D, et al. A systematic review and meta-analysis of the association between depression and insulin resistance. Diabetes Care. 2013;36(2): 480-9.

7. Ye J. Mechanisms of insulin resistance in obesity. Front Med. 2013;7(1):14-24.

8. Trirogoff ML, Shintani A, Himmelfarb J, IkizlerTA. Body mass index and fat mass are the primary correlates of insulin resistance in nondiabetic stage 3-4 chronic kidney disease patients. Am J Clin Nutr. 2007;86(6):1642-8.

9. Abbasi F, Malhotra D, Mathur A, Reaven GM, Molina CR. Body mass index and waist circumference associated to a comparable degree with insulin resistance and relatedmetabolic abnormalities in South Asian women and men. Diab Vasc Dis Res. 2012;9(4):296-300.

10. Takahara $M$, Kaneto $H$, Shimomura I. Insulin resistance can be easily estimated by body mass index and waist circumference in a general Japanese population. J Atheroscler Thromb. 2013;20(4):401-3.

11. Mamtani M, Kulkarni H, Dyer TD, Almasy L, Mahaney MC, Duggirala $R$, et al. Waist circumference independently associates with the risk of insulin resistance and type 2 diabetes in mexicanamerican families. PLoS One. 2013;8(3):e59153.

12. Krakauer NY, Krakauer JC. A new body shape index predicts mortality hazard independently of body mass index. PLoS One. 2012;7(7):e39504.

13. Krakauer NY, Krakauer JC. Dynamic association of mortality hazard with body shape. PLoS One. 2014;9(2):e88793.

14. He S, Zheng $Y$, Wang $H$, Chen $X$. Assessing the relationship between a body shape index and mortality in a group of middleaged men. Clin Nutr. 2016; pii: S0261-5614(16)31241-9. (Epub ahead of print)

15. Haghighatdoost F, Sarrafzadegan N, Mohammadifard N, Asgary S, Boshtam M, Azadbakht L. Assessing body shape index as a risk predictor for cardiovascular diseases and metabolic syndrome among Iranian adults. Nutrition. 2014;30(6):636-44.

16. Maessen MF, Eijsvogels TM, Verheggen RJ, Hopman MT, Verbeek $A L$, de Vegt $F$. Entering a new era of body indices: the feasibility of a body shape index and body roundness index to identify cardiovascular health status. PLoS One. 2014;9(9):e107212.

17. Eom BW, Joo J, Yoon HM, Ryu KW, Kim YW, Lee JH. A body shape index has a good correlation with postoperative complications in gastric cancer surgery. Ann Surg Oncol. 2014;21(4):1115-22.

18. Cheung YB. "A Body Shape Index" in middle-age and older Indonesian population: scaling exponents and association withincident hypertension. PLoS One. 2014; 9(1):e85421. 
19. Afsar B, Elsurer R, Kirkpantur A. Body shape index and mortality in hemodialysis patients. Nutrition 2013;29(10):1214-8.

20. Biolo G, Di Girolamo FG, Breglia A, Chiuc M, Baglio V, Vinci P, et al. Inverse relationship between "a body shape index" (ABSI) and fat-free mass in women and men: insights intomechanisms of sarcopenic obesity. Clin Nutr. 2015;34(2):323-7.

21. Sowmya S, Thomas T, Bharathi AV, Sucharita S. A body shape index and heart rate variability in healthy indians with low body mass index. J Nutr Metab. 2014;2014:865313.

22. Duncan MJ, Mota J, Vale S, Santos MP, Ribeiro JC. Associations between body mass index, waist circumference and body shape index with resting blood pressurein Portuguese adolescents. Ann Hum Biol. 2013;40(2):163-7.

23. Giudici KV, Martini LA. Comparison between body mass index and a body shape index with adiponectin/leptin ratio and markers of glucose metabolism among adolescents. Ann Hum Biol. 2017;44(6):489-94.

24. Behboudi-Gandevani S, Ramezani Tehrani F, Cheraghi L, Azizi F. Could "a body shape index" and "waist to height ratio" predict insulin resistance and metabolic syndrome in polycystic ovary syndrome? Eur J Obstet Gynecol Reprod Biol. 2016,205(10):110-4.

25. Rondanelli M, Klersy C, Perna S, Faliva MA, Montorfano G, Roderi $P$, et al. Effects of two-months balanced diet in metabolically healthy obesity: lipid correlations with gender and BMI-related differences. Lipids Health Dis. 2015,29(10)14:139.

26. Bonora E, Kiechl S, Willeit J, Oberhollenzer F, Egger G, Targher G, et al. Prevalence of insulin resistance in metabolic disorders: the Bruneck Study. Diabetes. 1998;47(10):1643-9.

27. Tabata S, Yoshimitsu S, Hamachi T, Abe H, Ohnaka K, Kono S. Waist circumference and insulin resistance: a cross-sectional study of Japanese men. BMC Endocr Disord. 2009 Jan 12;9:1.

28. Naderi N, Boobejame P, Bakhshandeh H, Amin A, Taghavi S, Male$\mathrm{ki} \mathrm{M}$. Insulin resistance in pulmonary arterial hypertension, is it a novel disease modifier? Res Cardiovasc Med. 2014;3(3):e19710.

29. McKeigue PM, Shah B, Marmot MG. Relation of central obesity and insulin resistance with high diabetes prevalence and cardiovascular risk in South Asians. Lancet. 1991;337(8738):382-6.

30. Lee $\mathrm{CH}$, Shih AZL, Woo YC, Fong CHY, Leung OY, Janus E, et al. Optimal Cut-Offs of Homeostasis Model Assessment of Insulin
Resistance (HOMA-IR) to Identify Dysglycemia and Type 2 Diabetes Mellitus: A 15-Year Prospective Study in Chinese. PLoS One. 2016;11(9):e0163424.

31. Banerji MA, Faridi N, Atluri R, Chaiken RL, Lebovitz HE. Body composition, visceral fat, leptin, and insulin resistance in Asian Indian men. J Clin Endocrinol Metab. 1999;84(1):137-44.

32. Chung JJ, Markiewicz MA, Poli囚 B, Shaw AS. Role of NKG2D in Obesity-Induced Adipose Tissue Inflammation and Insulin Resistance. PLoS One. 2014;9(10):e110108.

33. Zhang M, Zhang H, Wang C, Ren Y, Wang B, Zhang L, et al. Development and Validation of a Risk-Score Model forType 2 Diabetes: A Cohort Study of a Rural Adult Chinese Population. PLoS One. 2016;11(4):e0152054.

34. Stolk RP, Suriyawongpaisal P, Aekplakorn W, Woodward M, Neal $\mathrm{B}$; InterASIA Collaborative Group. Fat distribution is strongly associated with plasma glucose levels and diabetes in Thai adults - the InterASIA study. Diabetologia. 2005;48(4):657-60.

35. Lear SA, Humphries KH, Kohli S, Chockalingam A, Frohlich JJ, Birmingham CL. Visceral adipose tissue accumulation differs according to ethnic background: results of the Multicultural Community Health Assessment Trial (M-CHAT). Am J Clin Nutr. 2007;86(2):353-9.

36. DeFronzo RA, Tobin JD, Andres R. Glucose clamp technique: a method for quantifying insulin secretion and resistance. Am J Physiol Endocrinol Metab. 1979;237(3):E214.

37. Wallace TM, Matthews DR. The assessment of insulin resistance in man. Diabet Med. 2002;19:527-34.

38. GuanX, Sun G, Zheng L, Hu W, Li W, Sun Y. Associations between metabolic risk factors and body mass index, waist circumference, waist-to-height ratio and waist-to-hip ratio in a Chinese rural population. J Diabetes Investig. 2016;7(4):601-6.

39. He J, He S, Liu K, Wang Y, Shi D, Chen X. The TG/HDL-C Ratio Might Be a Surrogate for Insulin Resistance in Chinese Nonobese Women. Int J Endocrinol. 2014;2014:105168.

40. Gayoso-Diz P, Otero-Gonzalez A, Rodriguez-Alvarez MX, Gude F, Cadarso-Suarez C, García F, et al. Insulin resistance index (HOMA-IR) levels in a general adult population: curves percentile by gender and age. The EPIRCE study. Diabetes Res Clin Pract. 2011;94(1):146-55. 